\title{
Dosimetry estimation of SPECT/CT for iodine 123-labeled metaiodobenzylguanidine in children
}

\author{
Aida Mhiri, Ihsen Slim, Mohamed Faouzi Slimène \\ Department of Nuclear Medicine, Salah Azaiez Institute, Tunis, Tunisia
}

[Presented at the $4^{\text {th }}$ African Regional IRPA congress (AFRIRPA04), which was held from September 13-17, 2014 in Rabat, Morocco. This paper was reviewed and accepted by the scientific committee of the $4^{\text {th }} A$ frican Regional IRPA congress]

\section{Conference Proceeding}

\begin{abstract}
Purpose: To evaluate the additional radiation exposure in terms of effective dose incurred by patients in the CT (computed tomography) portion of ${ }^{123}$ I-MIBG (123II-metaiodobenzylguanidine) study with SPECT/CT (Single photon emission computed tomography associated to computed tomography) in some pediatric patients of our department. Methods: Data from 123II-MIBG scans comprising 50 children were presented in this study. The contribution of total effective dose imparted by the nuclear tracer and patient's age was calculated. Effective dose from the CT portion of the examination is also estimated.SPECT acquisitions were performed with a dual-headed SPECT unit with an integrated 2-slice CT scanner (Symbia T E-Cam, Siemens Medical Systems, Erlangen, Germany). The CT acquisition were performed using a tube current modulation system (Care Dose 4D). Parameters used were: tube current of $30-60 \mathrm{mAs}$, slice thickness of 3-5 mm, and tube voltage of $110 \mathrm{kV}$. Results: Our results show that SPECT dosimetry depends on administered activity and patient's age and weight. For CT scan, effective dose is affected by tube current $(\mathrm{mA})$, tube potential $(\mathrm{kVp})$, rotation speed, pitch, slice thickness, patient mass, and the exact volume of the patient that is being imaged. Conclusion: For children, 123II-MIBG study with SPECT/CT should be performed using the lowest available voltage and current. A sensible choice of these two parameters used can significantly reduce radiation dose, without any compromise in the quality of the diagnostic information.
\end{abstract}

Keywords: 123II-Metaiodobenzylguanidine; SPECT-CT- Pediatric; Dosimetry

\section{Introduction}

Metaiodobenzylguanidine (MIBG) is used for scintigraphic imaging of the adrenomedullary tumors pheochromocytoma and neuroblastoma in children. The use of I23I-l for labeling takes advantage of the better physical properties of I23I for imaging, allows higher activities to be administered with favorable radiation dosimetry and greater photon flux resulting in higher count, higher quality planar images and permits the performance of the single photon computed tomography (SPECT). ${ }^{1-3}$ Single photon emission computed tomography associated to X-ray computed tomography (SPECT/CT) is a nuclear medicine tomographic imaging technique which improves diagnostic accuracy for particular clinical indications due to the possible attenuation and/or scatter correction of the SPECT functional images and the availability of helpful anatomic information. ${ }^{4}$

Also, the interpretation of scintigraphic images can be confounded by physiological uptake, which can be better identified with SPECT/CT. However, it results in a significant increase of patient dose. In fact, according to literature data SPECT/CT compared to SPECT alone causes more radiation to the patient which is not sufficient to cause deterministic effects. 2, 4, 5, 6 For SPECT effective dose depends on administered activity and patient's age. The average radiopharmaceutical effective dose varies from tens to thousands of $\mathrm{mSv}$ for some nuclear medicine exams. ${ }^{4}$ However, the introduction of CT in nuclear diagnostic process results in a significant increase of the patient dose. In general, effective dose (E) for CT examinations can be higher than most other diagnostic imaging modalities ${ }^{6}$. Some authors have questioned the need to reduce doses particularly in children. ${ }^{7-8}$

Understanding radiation dosimetry and its potential for deleterious health effects, having knowledge of the magnitude of the effective dose and the dose to specific organs from SPECT and CT, and considering the role of CT in the context of SPECT/CT will allow the reader to reduce the radiation dose to the patient without compromising the quality of the patient's care. ${ }^{9}$ In this article, we will present the dosimetry associated with pediatric SPECT/CT for iodine 123-labeled metaiodobenzylguanidine, in terms of effective dose, in some pediatric patients of our department. Factors affecting the radiation dose associated with SPECT, using 123II-MIBG in children will be described. The dosimetry associated with 
the CT component will be also noted.

\section{Methods and Materials}

Data from 123II-MIBG scans comprising 50 children aged between 1 and 10 years, were presented in this study. The contribution of total effective dose imparted by the nuclear tracer and patient's age was calculated. Effective dose from the CT portion of the examination is also estimated.

SPECT acquisitions were performed with a dual-headed SPECT unit with an integrated 2-slice CT scanner (Symbia T E-Cam, Siemens Medical Systems, Erlangen, Germany). The CT acquisition were performed using a tube current modulation system (Care Dose 4D). Parameters used were: tube current of $30-60 \mathrm{mAs}$, slice thickness of 3-5 mm, and tube voltage of $110 \mathrm{kV}$.

For each child the CT was acquired immediately after SPECT; the patient being kept in the same position to minimize offsets due to movement and allow proper registration on fused imaging. The contribution of total effective dose imparted by the nuclear tracers for each child was calculated by multiplying the average administered activity for all patients by the "effective dose per unit administered activity" conversion factors listed in the International Commission on Radiological Protection (ICRP) Publication $53^{7}$ and $80{ }^{10-11}$. The effective dose from the CT portion of the examination is estimated from the product of the dose length product (DLP) and a body-region-specific conversion factor, $k\left(\mathrm{mSv} \mathrm{mGy}^{-1}\right.$ $\mathrm{cm}^{-1}$ ), which take into account the varying biological sensitivities of different organs as given in Table $1 .^{12}$

DLP is a patient-specific value determined by the scan length and the acquisition parameters; it represents the total amount of radiation delivered in the acquisition. CT scan was acquired immediately following completion of the SPECT study with the child in the same position to minimize motion errors.

\section{Results}

While for SPECT, dosimetry is dependent on administered activity and the patient's age and weight; for CT scan, there are many factors which affect dosimetry. Dose estimates (Table 2) are dependent on tube current (mA), tube potential $(\mathrm{kVp})$, rotation speed, pitch, slice thickness, patient mass, and the exact volume of the patient that is being imaged. According to the literature data, for children, MIBG imaging can be performed using the lowest available voltage and current.

\section{Discussion}

According to Gelfand ${ }^{13}$ and Fahey ${ }^{9}$, combined imaging results in a significant increase of the patient dose. Effective dose is directly dependent on administered activity and the patient's age for SPECT; whereas as described by Mhiri ${ }^{14}$, for CT scan, it depends on tube current, tube potential, rotation speed, pitch, slice thickness, patient mass, and the exact volume of the patient that is being imaged. A sensible choice of these parameters used, can significantly reduce radiation dose, without any compromise in the quality of the diagnostic information, according to Piwowarska ${ }^{15}$ and Larking ${ }^{5}$. The dose in the patient must be as low as compatible with the medical purpose. For Larkin ${ }^{5}$ and Valentin ${ }^{7}$, practice leading to a medical exposure must be clearly justified and protection against radiation must be optimized, particularly for children. Also, quality control procedures have to be defined because of the coupling between the two devices. ${ }^{16}$

TABLE 1: Factors for DPL/effective dose Conversion over various body regions and patient ages. ${ }^{12}$

\begin{tabular}{|c|c|c|c|c|}
\hline \multirow[t]{2}{*}{ Region of body } & \multicolumn{4}{|c|}{ Effective dose per DLP (mSv (mGy cm)-1) by age } \\
\hline & $<1$-year-old & 1-year-old & 5-year-old & 10 -year-old \\
\hline Head and neck & 0.013 & 0.0085 & 0.0057 & 0.0042 \\
\hline Head & 0.011 & 0.0067 & 0.0040 & 0.0032 \\
\hline Neck & 0.017 & 0.012 & 0.011 & 0.0079 \\
\hline Chest & 0.039 & 0.026 & 0.018 & 0,013 \\
\hline Abdomen and pelvis & 0.049 & 0.030 & 0.020 & 0.015 \\
\hline Trunk & 0.044 & 0.028 & 0.019 & 0.014 \\
\hline
\end{tabular}

TABLE 2: Effective doses (E) delivered by 123 I-MIBG SPECT-CT scintigraphy over patient ages and body regions.

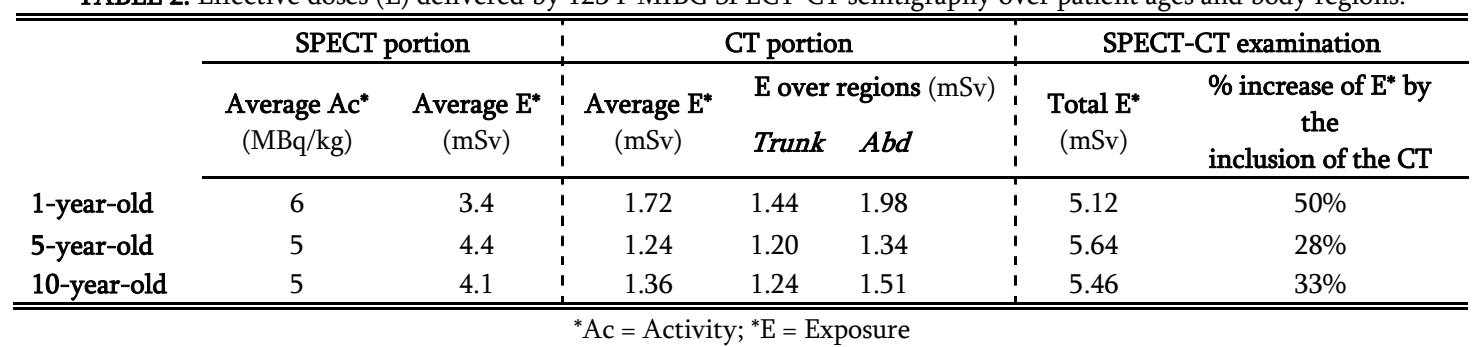




\section{Conclusion}

Compared to planer images, 123I-MIBG study with SPECT/CT increases significantly the number of lesions detected and allows better anatomic localization of neuroblastoma deposits and delineation of normal bowel activity. However, patient dose increases significantly also. Then, reducing the patient dose should be a constant preoccupation of prescribing physician, nuclear physician's and qualified personnel performing the act, particularly for child. Every effort should be made to adhere to the "As Low as Reasonably Achievable (ALARA)" principle and ensure that the patient is not subjected to unnecessarily high levels of radiation, still more at young age.

\section{Conflict of interest}

The authors declare that they have no conflicts of interest. The authors alone are responsible for the content and writing of the paper.

\section{References}

1. Shulkin BL, Shapiro B. Current concepts on the diagnostic use of MIBG in children. I Nucl Med 1998; 39:679-88.

2. Leung A, Shapiro B, Hattner R, et al. Specificity of radioiodinated MIBG for neural crest tumors in childhood. J Nucl Med 1997; 38:1352-7.

3. Rozovsky K, Koplewitz BZ, Krausz Y, et al. Added Value of SPECT/CT for Correlation of MIBG Scintigraphy and Diagnostic CT in Neuroblastoma and Pheochromocytoma. AJR Am J Roentgenol 2008; 190:1085-90.

4. Mhiri A, Slim I, Ghezaiel M, et al. L'apport de l'imagerie hybride TEMP/TDM dans la prise en charge du cancer différencié de la thyroïde. Médecine Nucléaire 2012; 36:554-60.

5. Larkin AM, Serulle Y, Wagner S, et al. Quantifying the increase in radiation exposure associated with SPECT/CT compared to SPECT alone for routine nuclear medicine examinations. Int $J$ Mol Imag 2011; 2011:897202.

6. Wall BF, Hart D. Revised radiation doses for typical X-ray examinations. Report on a recent review of doses to patients from medical X-ray examinations in the UK by NRPB. National Radiological Protection Board. Br J Radiol 1997; 70:437-9.

7. Valentin J. International Commission on Radiation Protection. Managing patient dose in multi-detector computed tomography (MDCT). ICRP Publication 102. Ann ICRP 2007; 37:1-79, iii.

8. The measurement, reporting, and management of radiation dose in CT. Report of AAPM Task Group 23, AAPM report no. 96, diagnostic Imaging
Council CT Committee 2008. Available from http://www.aapm.org/pubs/reports/RPT_96.pdf

9. Fahey FH. Dosimetry of Pediatric PET/CT. J Nucl Med 2009; 50:1483-91.

10. Valentin J. Radiation dose to patients from radiopharmaceuticals: (Addendum 2 to ICRP publication 53) ICRP publication 80 approved by the commission in September 1997. Annals of the ICRP 1998; 28: 1-126.

11. International Commission on Radiological Protection. Radiation dose to patients from radiopharmaceuticals. Oxford, Pergamon Press 1987. Publication 53; 18: 4.

12. Shrimpton PC, Hillier MC, Lewis MA, Dunn M. National survey of doses from CT in the UK: 2003. Br J Radiol 2006; 79:968-80.

13. Gelfand MJ, Lemen LC. PET/CT and SPECT/CT dosimetry in children: the challenge to the pediatric imager. Semin Nucl Med 2007; 37:391-8.

14. Mhiri A, Slim I, Ghezaiel M, et al. Estimation of Radiation Dosimetry for some Common SPECT-CT Exams. International Journal of Biotechnology for Wellness Industries 2012; 1: 266-9.

15. Piwowarska-Bilska H, Hahn LJ, Birkenfeld B, et al. Optimization of low-dose CT protocol in pediatric nuclear medicine imaging. J Nucl Med Technol 2010; 38:181-5.

16. Acquah GF, Schiestl B, Cofie AY, Nkansah JO. Radiation dose reduction without degrading image quality during computed tomography examinations: Dosimetry and quality control study. Int J Cancer Ther Oncol 2014; 2:02039. 